\title{
H ATOM OBSERVATIONS IN NEAR-STELLAR ENVIRONMENTS
}

\author{
LUIS F. RODRIGUEZ \\ Instituto de Astronomía, UNAM \\ Apdo. Postal 70-264 \\ México, DF 04510, México
}

\begin{abstract}
Interferometric observations of the 21-cm line of atomic hydrogen resolve out the emission from extended, line-of-sight clouds and allow the detailed study of compact $\mathrm{H}$ I structures in the surroundings of some stars. These atomic hydrogen components most probably are the result of photodissociation of gas that originally was in molecular form. They have been observed in H II regions, reflection nebulae, and planetary nebulae. The study of this atomic hydrogen component is important to determine the mass and physical conditions of gas in the environment of luminous stars and to test our theoretical knowledge of photodissociation regions.
\end{abstract}

\section{INTRODUCTION}

If a star of sufficiently high temperature is embedded in a molecular environment, it is expected that photodissociation of this environment will take place. A very important tracer of this photodissociated gas is the presence of atomic hydrogen, that could in principle be observed via its $21-\mathrm{cm}$ hyperfine transition. However, the actual observation of $\mathrm{H} \mathrm{I}$ in near-stellar environments is a difficult observational problem.

Consider, for example, observations made with a single dish with a beam size of $\mathbf{3 0}$ arc min, pointing in a direction that goes through $10 \mathrm{kpc}$ of $\mathrm{H} \mathrm{I}$ with a mean density of $1 \mathrm{~cm}^{-3}$. In the volume observed by the beam we will have of the order of $5 \times 10^{5} \mathrm{M}_{\odot}$ of atomic hydrogen. Since the mass of the H I associated with the object of interest could be as small as a fraction of a solar mass, it is obvious that the observer may have a hard time disentangling the origin of the observed emission.

The successful observation of $\mathrm{H} I$ in the nearby environment of a star requires of several conditions: i) an interferometer is needed to obtain high angular resolution and to establish the positions of the sources of emission, ii) the radial velocity of the $\mathrm{H} \mathrm{I}$ associated with the object studied should differ from that of large diffuse clouds in the same line of sight, and iii) preferably the source should be located away from the galactic plane where most of the $\mathrm{H}$ I exists. Under these favorable conditions it has been possible to study photodissociated $\mathrm{H} \mathrm{I}$ in association with planetary nebulae, $\mathrm{H}$ II regions, and reflection nebulae. 


\section{PLANETARY NEBULAE}

The first detection of $\mathrm{H} \mathrm{I}$ in planetary nebulae was made in NGC 6302 (Rodríguez and Moran 1982). Since then, $H$ I has been found in association with six other planetary nebulae (see Taylor et al. 1990). This atomic hydrogen is usually detected in absorption against the thermal continuum produced by the inner, ionized part of the planetary nebula. In most of the sources, the mass in $\mathrm{H} I$ is comparable with that present in the envelope in the form of ionized and/or molecular hydrogen, implying then an important correction to the mass of the planetary nebula. It is believed that the planetary nebula was originally molecular and that as the stellar nucleus becomes progressively hotter, photodissociation and ionization produced the $\mathrm{H} \mathrm{I}$ and $\mathrm{H}$ II zones.

Taylor et al. (1989) have been able to observe the H I associated with IC 418 both in absorption and in emission. From these observations they derived a total mass of circumnebular neutral hydrogen of $0.35 \mathrm{M}_{\odot}$ and a kinetic temperature in the range of 150 to $350 \mathrm{~K}$.

\section{H II REGIONS AND REFLECTION NEBULAE}

Interferometric observations made mainly during the last decade have determined that there is $\mathrm{H}$ I associated with several $\mathrm{H} I I$ regions (Roger and Pedlar 1981; Joncas et al. 1985). The H I is often located in broad intermediary zones between the ionized and molecular components. This atomic component is almost certainly due to photodissociation of $\mathrm{H}_{2}$ by the ultraviolet radiation from the exciting star(s).

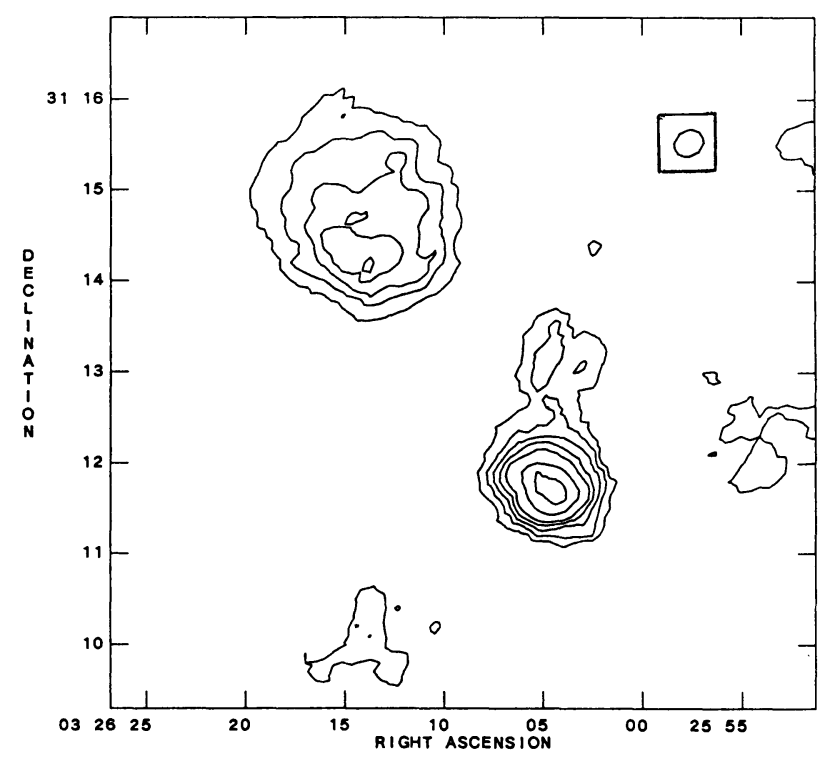

Figure 1. H I emission from the NGC 1331 region. The H I emission zones are approximately centered on $\mathrm{BD}+30^{\circ} 549$ (northeast) and SVS 3 (southeast), the illuminating stars of the reflection nebula NGC 1333. 
Using the Very Large Array, Rodríguez et al. (1990) detected H I in association with the reflection nebula NGC 1333. Two $\mathrm{H}$ I regions were detected, one associated with the star $\mathrm{BD}+30^{\circ} 549$, and the other with the star SVS 3 (Figure 1). Rodríguez et al. (1992) have observed these regions with greater angular and velocity resolutions and have been able to determine their mass and radius, which are given in Table 1.

Table 1

Parameters of Photodissociated H I Regions

\begin{tabular}{cccccc}
\hline Source & $\begin{array}{c}\mathrm{M}_{H I} \\
\left(\mathrm{M}_{\odot}\right)\end{array}$ & $\begin{array}{c}\text { Radius } \\
(\mathrm{pc})\end{array}$ & $\begin{array}{c}\dot{N}_{U V} \\
\left(\mathrm{~s}^{-1} \mathrm{~Hz}^{-1}\right)\end{array}$ & $\begin{array}{c}\mathrm{Sp} \\
(\mathrm{H} \mathrm{I})\end{array}$ & $\begin{array}{c}\mathrm{Sp} \\
(\text { Other })\end{array}$ \\
\hline BD+30 549 & 0.077 & 0.084 & $1.3 \times 10^{29}$ & $\mathrm{~B} 8$ & $\mathrm{~B} 9$ \\
SVS 3 & 0.057 & 0.042 & $1.3 \times 10^{30}$ & $\mathrm{~B} 6$ & $\mathrm{~B} 6$ \\
S187 & 70 & 1.2 & $2.0 \times 10^{32}$ & $\mathrm{~B} 1$ & $\mathrm{~B} 0$ \\
IC5146 & 450 & 3.5 & $1.9 \times 10^{32}$ & $\mathrm{~B} 1$ & $\mathrm{~B} 0$ \\
LkH $\alpha 101$ & 85 & 1.2 & $4.9 \times 10^{32}$ & B0 & B0 \\
\hline
\end{tabular}

In Figure 2 we show an $\mathrm{H}$ I spectrum taken at the center of the region associated with $\mathrm{BD}+30^{\circ} 549$. From the width of this line it is possible to set an upper limit to the kinetic temperature of the gas, $\mathrm{T}_{K} \leq 240 \mathrm{~K}$, while from the peak line brightness temperature it is possible to set a lower limit, $\mathrm{T}_{K} \geq 66 \mathrm{~K}$. Then, the gas in this photodissociated region has $66 \mathrm{~K} \leq \mathrm{T}_{K} \leq 240 \mathrm{~K}$. This range is in agreement with the values expected for a photodissociated region around a B-type star (Hollenbach et al. 1991).

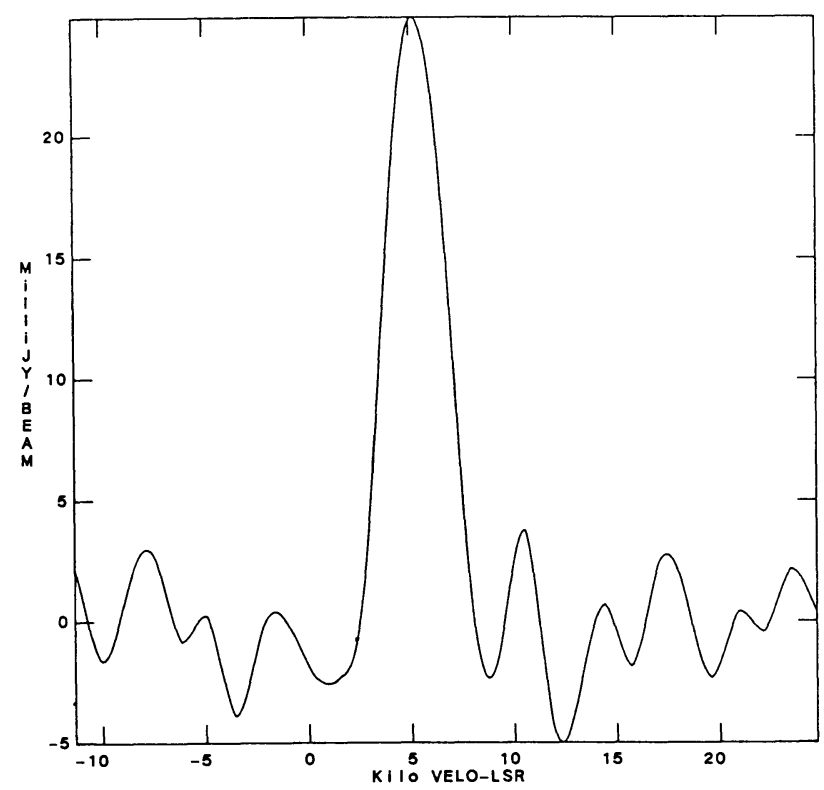

Figure 2. Spectrum of the $\mathrm{H} \mathrm{I}$ emission from the region associated with $\mathrm{BD}+30^{\circ} 549$. 
Knowing the mass and radius of the $\mathrm{H} I$ region, it is possible to estimate the rate of dissociating photons from numerical models of the photodissociated region around B-type stars (Escalante et al. 1992). These models have spherical symmetry, uniform density and gas temperature, and use the same parameters of the plane-parallel models of Sternberg and Dalgarno (1989). The results of the numerical models can be fitted to an expression of the form

$$
\left[\frac{\dot{N}_{U V}}{10^{30} s^{-1} H z^{-1}}\right]=0.73\left[\frac{M_{H I}}{0.01 M_{\odot}}\right]^{2}\left[\frac{R}{0.01 p c}\right]^{-3} \exp \left(4.70\left[\frac{M_{H I}}{0.01 M_{\odot}}\right]\left[\frac{R}{0.01 p c}\right]^{-2}\right),
$$

where $\dot{N}_{U V}$ is the photon rate per $\mathrm{Hz}$ produced by the central star at $1000 \AA, M_{H I}$ is the hydrogen mass, and $R$ is the radius of the region. From $\dot{N}_{U V}$, and asssuming a ZAMS star, one can determine the spectral type of the exciting star. In Table 1 we compare the spectral types derived from the $\mathrm{H} I$ observations with those derived by independent techniques for the $\mathrm{H}$ I regions associated with $\mathrm{BD}+30^{\circ} 549$, SVS 3 (Rodríguez et al. 1992), S187 (Joncas et al. 1992), IC5146 (Roger and Irwin 1982), and LkH $\alpha 101$ (Dewdney and Roger 1982). As can be seen, there is good agreement within one subclass.

\section{CONCLUSIONS}

Photodissociated $\mathrm{H}$ I regions have been observed in the 21-cm line of $\mathrm{H} \mathrm{I}$ in planetary nebulae, $\mathrm{H}$ II regions, and reflection nebulae. In the case of $\mathrm{H}$ II regions and reflection nebulae there is good agreement between observations and theory. Future observations of $\mathrm{H} I$ regions around deeply obscured, late B-type stars could provide a useful tool for the study of these stars that do not ionize significantly their surroundings, but that do produce detectable photodissociation.

\section{ACKLOWLEDGEMENTS}

I am indebted to J. Cantó, V. Escalante, and S. Lizano for valuable comments.

\section{REFERENCES}

Dewdney, P. E. and Roger, R. S. 1982, Ap. J., 255564.

Escalante, V., Lizano, S., Cantó, J., and Rodríguez, L. F. 1992, in preparation.

Hollenbach, D. J., Takahashi, T., and Tielens, A. G. G. M. 1991, Ap. J., 377, 192.

Joncas, G., Dewdney, P. E., Higgs, L. A., and Roy, J. R. 1985, Ap. J., $\underline{298,596 .}$

Joncas, G., Durand, D., and Roger, R. S. 1992, in preparation.

Rodríguez, L. F. and Moran, J. M. 1982, Nature, 239, 323.

Rodríguez, L. F., Lizano, S., Cantó, J., Escalante, V., and Mirabel, I. F. 1990, Ap. J. $\underline{365}, 261$.

Rodríguez, L. F., Lizano, S., Cantó, J., and Escalante, V. 1992, in preparation.

Roger, R. S. and Pedlar, A. 1981, Astron. Astrophys., $94,238$.

Roger, R. S. and Irwin, J. A. 1982, Ap. J., 256, 127.

Sternberg, A. and Dalgarno, A. 1989, Ap. J., $\underline{338}, 197$.

Taylor, A. R., Gussie, G. T., and Goss, W. M. 1989, Ap. J., 340, 932.

Taylor, A. R., Gussie, G. T., and Pottasch, S. R. 1990, Ap. J., $\underline{351}, 515$. 


\section{QUESTIONS AND ANSWERS}

J.C.Pecker: 1) Any Be star observed in your survey? 2) Have you detected (and measured) stellar winds around the B stars of your survey?

L.F.Rodriguez: 1) No, we do not have Be stars in our survey. Perhaps the Herbig Be stars, being associated with clouds, are good candidates to have $\mathrm{HI}$ regions around. 2) We do not detect any obvious effects of a wind in our data.

J.A.de Freitas Pacheco: We observe for PN an anti-correlation between $M_{H I I}$ x electron density, which can be explained by the propagation of an ionization front through the ejected envelope. Thus we would expect that the neutral $\mathrm{H}$ mass would decrease with time. Is there any correlation between $M_{H I}$ and kinematical age?

L.F.Rodriguez: Yes, you are right. Indeed, Taylor et al. (1990, Ap.J.,351, 515) do find an anticorrelation between $\mathrm{HI}$ optical depth and radius that could point to an anticorrelation between $\mathrm{HI}$ mass and age. 